\title{
Investigating Factors That Affect the Adoption of COVID-19 Contact-Tracing Apps: A Privacy Calculus Perspective
}

\author{
Yannic Meier, Judith Meinert, and Nicole C. Krämer \\ Social Psychology: Media and Communication, Department of Computer Science and Applied Cognitive Science, University of \\ Duisburg-Essen
}

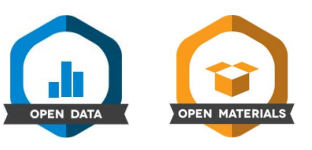

On June 16, 2020, the German government issued a coronavirus disease 19 (COVID-19) contact-tracing app whose privacy settings were fiercely debated in advance. Contrary, potential benefits regarding the acceleration of contact tracing were advertised. This study investigated the relative importance of privacy concerns and perceived benefits in relation to the decision to use the app adopting a privacy calculus perspective extended by trust in app privacy and technological knowledge about the app. Results of a representative German sample $(N=952)$ revealed that privacy concerns were negatively related to app usage, and perceived benefits and knowledge were positively related to actual app adoption. Additionally, perceiving app benefits was the most important factor for (continued) usage intention, while privacy concerns did not play a role anymore. Trust was strongly negatively associated with privacy concerns and strongly positively with perceived benefits. Results are discussed concerning consequences for communication strategies when launching technology whose adoption should help in mitigating dangers of pandemics.

Keywords: COVID-19 contact-tracing app, privacy calculus, perceived benefits, trust, factual knowledge

Supplemental materials: https://doi.org/10.1037/tmb0000040.supp

The coronavirus disease 19 (COVID-19) pandemic is the greatest global health disaster since the so-called Spanish flu 100 years ago. In the years of 1918-1920, about 50 million people died as a result of the

Action Editor: C. Shawn Green was the action editor for this article.

ORCID iDs: Yannic Meier (D) https://orcid.org/0000-0002-5726-3229; Judith Meinert (D) https://orcid.org/0000-0002-7387-197X; Nicole C. Krämer (iD https://orcid.org/0000-0001-7535-870X.

We have no conflicts of interest to disclose.

This work has been funded by the Federal Ministry for Education and Research of Germany (No. 16KIS0743). We acknowledge support by the Open Access Publication Fund of the University of Duisburg-Essen. This manuscript features online material including the items of the study, the $\mathrm{R}$ code, the dataset, and additional analyses: https://osf.io/turbs/.

Open Science Disclosures:

II The data are available at https://osf.io/turbs/

(1) The experiment materials are available at https://osf.io/turbs/

Open Access License: This work is licensed under a Creative Commons Attribution-NonCommercial-NoDerivatives 4.0 International License (CC-BYNC-ND). This license permits copying and redistributing the work in any medium or format for noncommercial use provided the original authors and source are credited and a link to the license is included in attribution. No derivative works are permitted under this license.

Disclaimer: Interactive content is included in the online version of this article.

Contact Information: Correspondence concerning this article should be addressed to Yannic Meier, Social Psychology: Media and Communication, Department of Computer Science and Applied Cognitive Science, University of Duisburg-Essen, Forsthausweg 2, 47057 Duisburg, Germany. Email: yannic.meier@uni-due.de virus disease (Johnson \& Mueller, 2002), thus having far more casualties than the First World War (Broadberry \& Harrison, 2005). In order to prevent similar numbers of victims in the current pandemic which would be exorbitantly high when transferred to todays' global population, several protective measures are recommended by official organizations, institutes, and scientists. Useful recommendations include keeping distance to other persons, wearing face masks in public places, and regularly washing or disinfecting one's hands (World Health Organization, 2020). Whereas these protective measures were already recommended 100 years ago, technological progress of the last decades allows us to employ new methods to contain the pandemic. Drastic examples are CCTV systems equipped with facial recognition abilities that could identify blacklisted persons who have tested positive for COVID-19 and violate quarantine obligations (Lin \& Hou, 2020) or big data techniques that are used to retrace infection chains on the basis of smartphone-generated location data. However, these approaches interfere with personal autonomy and privacy and are therefore ethically questioned (Mello \& Wang, 2020). One method that is privacy preserving, yet, at the same time efficient in retracing infection chains is contact tracing via a smartphone app (Yasaka et al., 2020). This kind of technology, however, can only efficiently support contact tracing and help to discontinue chains of infections when a critical number of citizens adopt the technology. A contact-tracing app may contain the pandemic when approximately $60 \%$ of a country's population use it, but also smaller numbers of users can have positive effects (Ferretti et al., 2020). Therefore, every individual who can be won to use the app is important to increase its usefulness and reach mass acceptance (cf. Trang et al., 2020). This entails the necessity for governments to promote the app's benefits and transparently disclose all possible costs 
that can arise by its usage, so that users can make an informed choice based on a cost-benefit analysis.

A theoretical lens that addresses such a privacy cost-benefit analysis is the privacy calculus (Culnan \& Armstrong, 1999). This approach assumes privacy costs and perceived benefits to impact privacyrelevant behaviors (e.g., technology adoption and usage). Based on this theoretical background and extending prior studies which merely focused on hypothetical scenarios (e.g., Kaptchuk et al., 2020; Walrave et al., 2020), we scrutinize factors influencing the decision to use the German COVID-19 contact-tracing app that was issued in Germany on June 16, 2020. To better understand the psychological factors that positively or negatively affect actual usage of the app as well as future usage intention of users and nonusers, we conducted a panel study $(N=952)$ in the week after the app's release. We were interested in the question whether the adoption of the German COVID19 contact-tracing app was based on a privacy calculus decision as well as trust toward and knowledge about the app.

\section{Theoretical Background}

\section{"Corona-Warn-App"}

Different forms of contact-tracing apps can be realized, but the most privacy-preserving possibility is that the app does not access and process the location of its users but uses Bluetooth to merely recognize and record when two smartphones that installed the app are in close proximity. With the purpose of identifying infection chains, the German contract-tracing app basically uses these anonymous codes via Bluetooth network that are stored locally and changed every 10-20 min. If users receive a notification that they had contact with another user who has tested positive for COVID19 , they must autonomously and voluntarily get tested, enter the test result into the app, and go into quarantine (for more information, please see: https://www.coronawarn.app/en/).

\section{Privacy Calculus}

The privacy calculus (Culnan \& Armstrong, 1999) assumes that people weigh the anticipated privacy costs and benefits before they decide to disclose personal information (Dienlin \& Metzger, 2016), install an app (Eling et al., 2013), or adopt new technology (Princi \& Krämer, 2020). For instance, perceived privacy costs (e.g., privacy threats) have been found to negatively affect online self-disclosure (Bol et al., 2018) or the likelihood to install an app (Eling et al., 2013). Contrary, persons who perceived benefits of website or app usage disclosed more personal data (Meier, Schäwel, \& Krämer, 2020) or had a higher intention to install an app (Krasnova et al., 2014). Some studies already investigated the privacy calculus in relation to mobile app usage. In general, privacy calculus notions have been found when it comes to people's intentions (Keith et al., 2016) and actual behaviors (Eling et al., 2013) to install everyday mobile applications. These studies indicate that an app's privacy issues and benefits are integrated in the decision to use an app or not. Moreover, it seems that the privacy calculus may also hold regarding COVID-19 contact-tracing apps. Hassandoust et al. (2021) found that persons with higher privacy risk beliefs had a lower intention to use a COVID-19 contact-tracing app and those who perceived the app to be beneficial had an increased usage intention. This study, however, did not investigate people's actual adoption behaviors of an existing tracing app. Hence, it is still an open question whether a weighing of privacy risks and benefits takes place when it comes to the use of an actual COVID app.

\section{The Influence of Benefits}

Benefits can be described as advantages resulting from an action such as the use of a technology (e.g., in consumer-related contexts receiving personalized recommendations and high-quality services) for which, in return, an access to personal data must be provided (Culnan \& Armstrong, 1999). This means that people must expect a positive outcome from an action to be motivated to perform it. Several studies found that the anticipation of social benefits increases the likelihood of information disclosure on social networking sites (SNSs; Krasnova et al., 2010) or that the perception of convenience increases likelihood of smart technology adoption (Zheng et al., 2018). Frequently, benefit perceptions are found to be the driving factor of self-disclosure or technology adoption (e.g., Dienlin \& Metzger, 2016; Krasnova et al., 2014; Princi \& Krämer, 2020). One explanation for this observation can be found in the gratification hypothesis that assumes the expected benefits to outweigh the perceived risks, for instance, because people overrate the gratifications or because they are unaware of the privacy threats (Trepte et al., 2015). With regard to COVID contact-tracing apps, a study addressing usage intentions of a hypothetical COVID-19 contact-tracing app in Belgium found perceived individual (e.g., get an information about being infected with COVID-19) and social benefits (e.g., support the identification of infection chains) to be the most important predictors (Walrave et al., 2020). Another study found that usage intention increases when people think that a hypothetical app can help to decrease the number of COVID-19 cases and it works accurately (Kaptchuk et al., 2020). In particular, individuals who are critical or undecided in terms of adopting the app seem to be mostly convinced to use such an app by benefit appeals (Trang et al., 2020). Here, it must be noted that the nature of COVID-19 contract-tracing app benefits differs from other benefits that are typically investigated. Benefits of using services and apps are particularly serving individual desires and interests (e.g., entertainment, social connectivity, or convenience), whereas advantages stemming from the adoption of tracing apps may include protecting oneself but are primarily focused on protecting others and society. In the long run, when numbers of new infections decrease one would certainly again benefit from mass acceptance of contact-tracing apps (cf. Trang et al., 2020).

The studies that have been conducted on the benefits of COVID19 contact-tracing apps so far rely on hypothetical scenarios which are known for their methodological deficiencies. Thus, the present study is one of the first attempts to scrutinize the factors influencing the decision to adopt an app that is actually available.

\section{Perceived Privacy Costs}

Besides benefits, the perceived privacy costs (e.g., privacy risks or concerns) determine whether a technology is adopted. Indeed, numerous studies indicate an influence of privacy concerns on the way technology is used (Utz \& Krämer, 2009; Zheng et al., 2018). However, research on SNSs has largely demonstrated that perceived benefits are more important predictors compared to perceived privacy costs (Bol et al., 2018; Khan et al., 2014; Meier, Schäwel, Kyewski, \& Krämer, 2020). Based on their finding that 
expected benefits have more predictive power regarding Facebook self-disclosure than privacy concerns, Dienlin and Metzger (2016) conclude that at least regarding SNSs "benefits loom larger than concerns" (p. 12). This finding might, however, not transfer to the adoption of a COVID-19 contact-tracing app. The reasons might be twofold: First, the benefits are not comparable to the individual, hedonistic benefits promised by disclosing data on SNSs. Instead, the benefits are more distal, societal gains which even do not pose a direct health advantage for users themselves (i.e., the app does not preclude infection but only enables to report one's own infection so that other people know that they might be infected). Second, the privacy aspects of the app have been fiercely debated before it was launched (Horn, 2020). Although eventually also NGOs well known for their data protection stance (Chaos Computer Club) endorsed the app, many laypeople will first and foremost associate the app with a fear of privacy risks. First studies with hypothetical scenarios also confirm that privacy concerns play an important role: A recent study found that persons who had privacy concerns toward a hypothetical COVID-19 contact-tracing app had a reduced usage intention, whereas participants who reported concerns toward the virus disease had an increased willingness to use the app (Trang et al., 2020). Here, concerns showed to have a stronger correlation with usage intention than COVID-19 anxiety. Other studies found negative relations between privacy concerns or privacy risk beliefs and COVID-19 tracing-app adoption intentions, too (Chan \& Saqib, 2021; Hassandoust et al., 2021; Kaptchuk et al., 2020).

Following the logic of the privacy calculus, people should weigh the potential benefits and privacy risks before they decide to use a contact-tracing app - although the relative importance of benefits and privacy concerns is unclear. Extending the results of first studies on hypothetical apps, we capitalized the opportunity to assess data on actual usage shortly after the launch of the German COVID-19 contact-tracing app. In a representative sample of German smartphone users, we were therefore able to investigate both people who have already adopted the app and people who have not (yet). We assume that people who have privacy concerns less often use the app, whereas those who perceive high benefits more frequently use it. The following hypotheses are derived:

H1: Privacy concerns will be negatively related to the usage of the contact-tracing app.

$H 2$ : Perceived benefits will be positively related to the usage of the contact-tracing app.

Because the app was only issued a week before the survey started, people may be intended to install the app although they have not done so. Others may want to deinstall the app again and only installed it because they were curious. Hence, we also assess people's future usage intentions independent of whether people have already installed the app or not. The following hypotheses are formulated:

$H 3$ : Privacy concerns will be negatively related to future usage intention.

H4: Perceived benefits will be positively related to future usage intention.

\section{Trust in App Privacy}

Media reports have frequently assumed that trust will play an essential role for the adoption of the COVID-19 contact-tracing app (Horn, 2020). Trust can be described as an expectation of another party's behavior although the actual intention is unknown (Bhattacharya et al., 1998). In the original privacy calculus literature, trust had a positive influence on one's intention to share personal data (Culnan \& Armstrong, 1999; Dinev \& Hart, 2006). Further studies have shown that trust can mitigate perceived privacy risks (Krasnova et al., 2010) and can indirectly positively contribute to one's intention to adopt a fitness app (Beldad \& Hegner, 2018). The latter study found trust toward the provider of the fitness app to increase participants' perceived usefulness of the app which was then positively related to usage intention. Regarding COVID-19 tracing apps, a recent study found that people who trusted the government had a higher adoption intention (Altmann et al., 2020). In the present study, trust will be conceptualized in terms of privacy and is defined as the belief that the app developers have honestly communicated any privacy issues and have done their very best to guarantee the highest possible privacy protection. With respect to the German COVID-19 contact-tracing app, the official providers are the Robert Koch Institute (the government's central scientific institution in the field of biomedicine and infectious diseases) and the German government. Hence, we assume that people who trust the providers of the COVID-19 contact-tracing app will have decreased privacy concerns and an increased perception of the app's benefits. The following hypotheses are derived with regard to actual usage:

H5: Trust will be positively related to the usage of the app.

With regard to usage intention independent of whether people have already installed the app, we assume:

H6: Trust will be positively related to future usage intention.

H7: Trust will be negatively related to privacy concerns.

H8: Trust will be positively related to perceived benefits.

\section{Knowledge About Privacy Features}

Prior to the release of the COVID-19 contact-tracing app in Germany, a major governmental information campaign has been launched. Its objective was to clearly communicate the advantages of this app regarding the containment of the virus disease and to convince people that privacy concerns are unfounded as the application can only assess anonymized codes that cannot be traced back to individuals. A recent study found that higher knowledge about a privacy-friendly SNS was related to increased perceptions of privacy on that website (Meier, Schäwel, \& Krämer, 2020). Hence, more knowledge about the functioning of the COVID-19 contacttracing app should lead to decreased privacy concerns. Moreover, the same study found that a website that is portrayed to be privacy preserving is perceived as more beneficial than the same website that is described as privacy invading (Meier, Schäwel, \& Krämer, 2020). Concerning COVID-19 tracing apps, a recent study found that persons who believed to understand how an app works were more willing to use it (Kostka \& Habich-Sobiegalla, 2020). However, this measurement focused on a subjective rather than an 
objective assessment of one's knowledge. Consequently, it can be assumed that a higher objective knowledge that the contact tracing app respects privacy is positively related to the intention to use it and to the appreciation of the app's benefits. Finally, people who know more about the privacy-friendliness of the app should also have more trust toward the app publishers. Therefore, we hypothesize the following with regard to actual usage:

H9: Knowledge about the privacy features of the app will be positively related to the usage of the app.

With regard to future usage intention, we assume:

H10: Knowledge about the privacy features of the app will be positively related to future usage intention.

H11: Knowledge about the privacy features of the app will be negatively related to privacy concerns.

H12: Knowledge about the privacy features of the app will be positively related to perceived benefits.

H13: Knowledge about the privacy features of the app will be positively related to trust.

\section{Method}

\section{Sample}

The present study is part of a longitudinal project with three waves of measurements. In the present study, only the first measurement point is being analyzed which was collected from June 22 to July 3, 2020, the week after the COVID-19 contact-tracing app was launched in Germany. A professional online panel provider was commissioned that recruited participants based on fixed quotas regarding sex and age distribution to achieve representativeness of German smartphone users between 18 and 74 years $(n=939)$. In addition, we advertised the study on Twitter $(n=13)$. In total, 952 persons participated in the online survey having a mean age of 43.8 years (range: $18-73 ; S D=14.1$ ). Four hundred and seventy-two participants were female and 480 were male. The sample was rather highly educated with 340 participants holding a university degree and 226 with high school graduation. Most of the participants were employed with 176 persons working in the technical field, 88 in the medical sector, and 259 had an occupation related to data protection issues. With regard to the use of the COVID-19 contact-tracing app,
366 individuals indicated to use the app, while 586 persons stated to not use the app (yet). Within the sample, 195 participants indicated that they know a person who has tested positive for COVID-19, whereas 757 do not know anyone who has fallen ill. The procedure of the study was approved by the local ethics committee.

\section{Measure}

The scales appear in the same order as they were presented in the survey and were assessed on 7-point Likert scales ( 1 = I do not agree at all to $7=$ I totally agree) unless stated differently. All items that were used in the current analysis were tested for factor validity in confirmatory factor analyses (CFAs). Measures of reliability and internal consistency can be viewed in Table 1. Moreover, we tested whether the results of the present study were biased by common method variance (CMV) because all data stem from the same source (i.e., a survey; Podsakoff et al., 2012). To do so, we extended a CFA containing the independent variables that were measured on the same scale (i.e., trust, perceived benefits, and privacy concerns) by an unmeasured latent method factor (ULMF) that predicts all measured variables (i.e., items). To evaluate whether CMV may have affected the results, we compared the correlations of the variables not controlled for the ULMF to those controlled for the ULMF. The uncontrolled correlations were minimally higher compared to the controlled ones. Thus, we conclude that the findings of the present study are only marginally biased by CMV (Podsakoff et al., 2012). All items (both German and English) and the results of the CMV control can be seen in the OSF (https://osf.io/turbs/; Meier et al., 2021).

To assess participants' factual knowledge about the app and how it works, 10 statements which were either false or true (with the answering options wrong, right, or I don't know) concerning the COVID-19 contact-tracing app had to be answered. Statements were taken from the official information website (https://www.coronawarn .app/en/). Wrong answers and the "I don't know" option were coded as zero and correct answers were coded as one. Thus, higher values indicate a higher level of knowledge. Respondents were asked if they already use the COVID-19 contact-tracing app. If they responded that they already use it, they were asked to indicate if they want to continue using the app with three items. If they answered that they do not use the app, they were asked about their usage intentions in the future with three items. For the path model, one score combining the future usage intention of users and nonusers was calculated. Perceived benefits of using the app were assessed with eight items. Based on a confirmatory factor analysis, a single-factor solution was computed. To measure

Table 1

Results of the Confirmatory Factor Analyses

\begin{tabular}{|c|c|c|c|c|c|c|c|c|c|c|}
\hline Measured constructs & $\chi^{2}$ & $d f$ & $p$ & CFI & TLI & RMSEA & SRMR & $\alpha$ & $\omega$ & AVE \\
\hline Privacy concerns ${ }^{\mathrm{a}}$ & 710.08 & 27 & $<.001$ & .94 & .92 & .16 & .08 & .96 & .95 & .75 \\
\hline Perceived benefits $^{\mathrm{a}}$ & 353.04 & 20 & $<.001$ & .96 & .94 & .13 & .04 & .97 & .97 & .80 \\
\hline Trust $^{\mathrm{a}}$ & 22.48 & 5 & $<.001$ & 1.00 & .99 & .06 & .01 & .97 & .97 & .88 \\
\hline Knowledge $^{\mathrm{b}}$ & 152.96 & 35 & $<.001$ & .98 & .98 & .06 & .07 & .91 & .84 & .54 \\
\hline Usage intention (users) ${ }^{\mathrm{c}}$ & - & - & - & - & - & - & - & .71 & .71 & .45 \\
\hline Usage intention (nonusers) ${ }^{c}$ & - & - & - & - & - & - & - & .83 & .90 & .77 \\
\hline
\end{tabular}

Note. Reliability measures are Cronbach's $\alpha$, McDonald's $\omega$, and average variance extracted. CFI = comparative fit index; TLI = Tucker-Lewis index; RMSEA = root-mean-square error of approximation; SRMR = standardized root-mean-square residual; AVE = average variance extracted.

${ }^{a}$ Maximum likelihood estimation and Satorra-Bentler correction. ${ }^{\mathrm{b}}$ Diagonally weighted least squares estimation. ${ }^{\mathrm{c}}$ Model is saturated. 
participants' trust in the app operators, five items had to be rated. Based on a confirmatory factor analysis, a single-factor solution was yielded. For the assessment of participants' privacy concerns, nine items were used. Confirmatory factor analysis indicated a singlefactor solution.

\section{Power Analysis}

In order to determine the minimum detectable effect size (MDE; cf. Djimeu \& Houndolo, 2016) that can still be meaningfully interpreted given our current sample size and analytical method, we calculated different simulation analyses using the structure of the two structural equation models (SEMs). Power levels of at least $90 \%$ are desirable (Curran-Everett, 2017). Power analyses for latent variable SEMs not only depend on parameters like effect size, power, and $\alpha$ level but also on model complexity and reliability of the latent constructs (Wang \& Rhemtulla, 2021). We included different (small to large) effect sizes into the simulations and varied the reliability of the latent factors while keeping the $\alpha$ level $(\alpha=.05)$ and sample size $(N=952)$ constant. We ran 1,000 replications for each model. For a detailed overview, please see the OSF material (https://osf.io/turbs/; Meier et al., 2021). Power to find very small effects increased with increasing reliability. Given the fact that all scales achieved high reliability in the present study, it can be concluded that both models can detect effects as small as $\beta=\mathrm{I} .10 \mathrm{l}$ with a probability of more than $90 \%$. Hence, we set the MDE to $\beta=|.10|$ and do not interpret effects smaller than that. Note that we refrained from calculating post hoc power using the exact model parameters because calculating post hoc power does not provide any additional information (cf. Lakens, 2021). Hence, we can only report approximate values of achieved power rather than exact power levels.

\section{Results}

All statistical analysis were performed using $\mathrm{R}$ (version 4.0.3). Two SEMs with latent factors and diagonally weighted least squares (DWLS) estimation were run. The first model focused on the actual use of the app. The second model tested the hypotheses regarding participants' usage intention. Bivariate correlations can be seen in Table 2.

\section{Actual Usage Behavior}

Altogether, 366 participants stated they were currently using the contact-tracing app, while 586 respondents stated they were not. First, we analyzed whether privacy concerns, perceived benefits, trust, and app knowledge would be related to the app usage. We used structural equation modeling to test the direct relationships between the independent variables and app usage. The model is depicted in Figure 1. We modeled the independent factors as latent variables. DWLS estimation was used because there were binary endogenous variables in the model. The model produced a good model fit: $\chi^{2}(486)=769.75, p<.001, \chi^{2} / d f=1.58$, CFI $=.99$, TLI $=.99$, $\mathrm{RMSEA}=.03,90 \%$ CI $[.02, .03], \mathrm{SRMR}=.05$. However, it must be noted that the typically used fit indices tend to be inflated using the DWLS estimation which increases the risk of accepting misspecified models (Xia \& Yang, 2019).

The first hypothesis (H1) predicted a negative relation between privacy concerns and the app usage. Indeed, there was a negative relationship between privacy concerns and app usage $(\beta=-.19, p<$ .001) confirming H1. The second hypothesis (H2) was also supported by the data as there was a positive association between perceiving benefits and using the app $(\beta=.57, p<.001)$. Further, it was hypothesized that people who have trust in the app and its developers are more likely to use it (H5). This hypothesis, however, must be rejected as there was no relationship between trust and app usage $(\beta=-.04, p=.698)$. Finally, H9 was supported by the positive relation of knowledge about the app's privacy features and app usage $(\beta=.36, p<.001)$. Seventy-nine percent of variance in app usage was explained by the variables.

\section{Usage Intention}

The hypotheses regarding the intention (to continue) to use the COVID-19 contact-tracing app were also analyzed in a SEM using DWLS estimation and latent variables. Note that we had to use a manifest variable for app usage intention since the items for users and nonusers were slightly different. The model can be seen in Figure 2. Testing the model resulted in a good model fit: $\chi^{2}(486)=$ 703.94, $p<.001, \chi^{2} / d f=1.45, \mathrm{CFI}=1.00, \mathrm{TLI}=1.00, \mathrm{RMSEA}=$ $.02,90 \%$ CI $[.02, .03]$, SRMR $=.05$. Hypothesis H3 assuming that privacy concerns would be negatively related to the willingness to use the app in the future was not supported $(\beta=-.05, p=.433)$. The next hypothesis (H4) focused on the relation between perceived benefits and usage intention. Results found support for the assumption that people who perceived benefits from using the app would be more intended to use it $(\beta=.60, p<.001)$. The next three hypotheses focused on the relationships between trust and the privacy calculus as well as usage intention. H6 assuming that trust would be positively associated with the intention to use the app was not supported ( $\beta=.12, p=.486)$. In contrast, trust was negatively related to privacy concerns $(\beta=-.60, p<.001)$ and positively to benefit perception $(\beta=.80, p<.001)$ supporting hypotheses $\mathrm{H} 7$ and $\mathrm{H} 8$. The final three hypotheses targeted knowledge about the contact-tracing app. H10 that predicted a positive

Table 2

Means, Standard Deviations, and Bivariate Correlations of the Measured Constructs

\begin{tabular}{|c|c|c|c|c|c|c|}
\hline Measured constructs & $M(S D)$ & 1 & 2 & 3 & 4 & 5 \\
\hline 1 Privacy concerns & $3.1(1.8)$ & - & & & & \\
\hline 2 Perceived benefits & $4.9(1.7)$ & $-.50 * * *$ & - & & & \\
\hline 3 Trust & $4.8(1.9)$ & $-.66^{* * * *}$ & $.80^{* * *}$ & - & & \\
\hline 4 Knowledge & $5.7(2.9)$ & $-.41^{* * *}$ & $40^{* * *}$ & $.46^{* * *}$ & - & \\
\hline 5 App usage intention & $4.3(2.5)$ & $-.48^{* * *}$ & $.76^{* * *}$ & $.69^{* * *}$ & $.41^{* * *}$ & - \\
\hline 6 App usage $(0=$ no, $1=$ yes $)$ & $0.4(0.5)$ & $-.48^{* * *}$ & $.59^{* * *}$ & $.56^{* * *}$ & $.43^{* * *}$ & $.70^{* * * *}$ \\
\hline
\end{tabular}

$* * p<.001$. 
Figure 1

Structural Equation Model With App Usage as Endogenous Variable

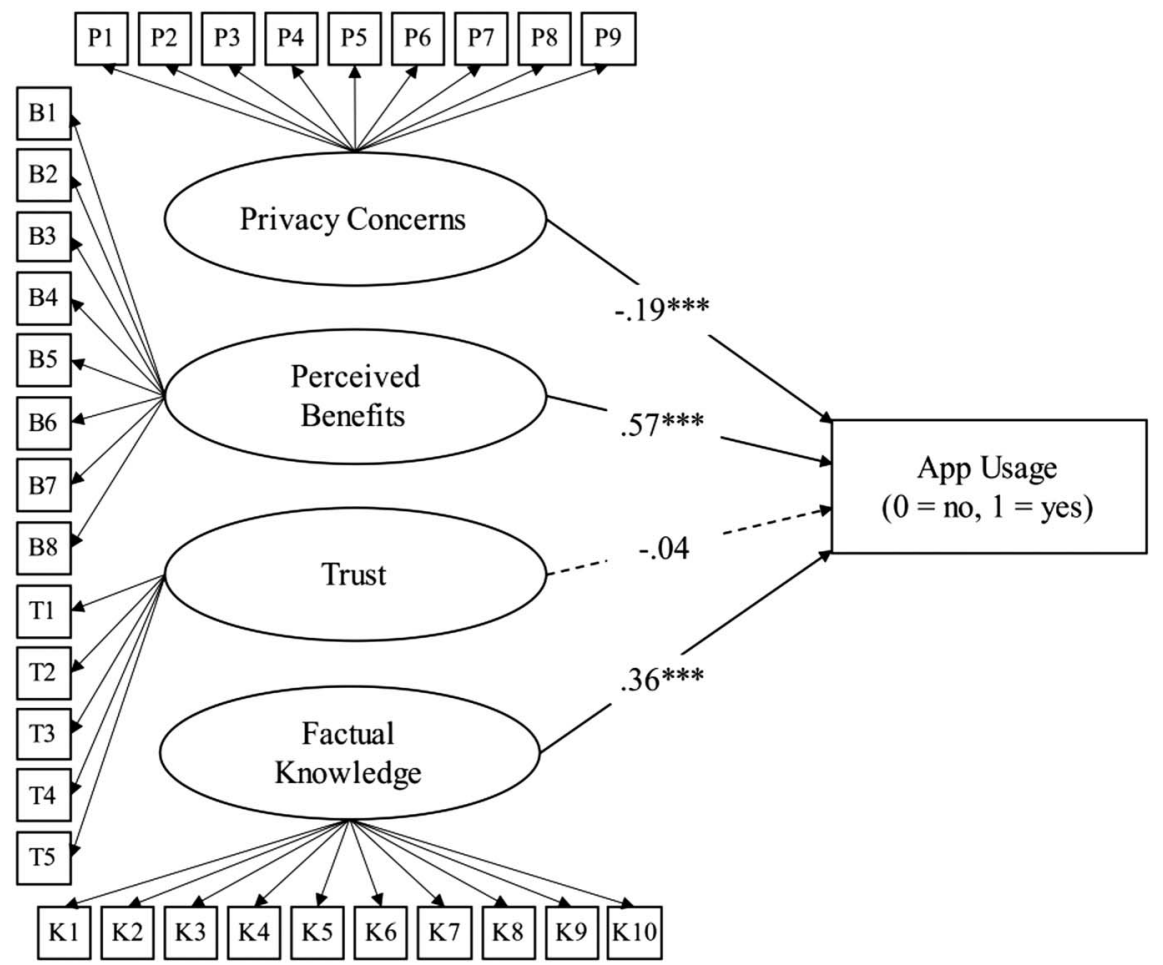

Note. Numbers represent standardized regression coefficients. Dotted line indicates that the path is not significant. Note that the latent variables are correlated which is not depicted in the figure due to better clarity.

$* * * p<.001$.

relation between knowledge and usage intention and results revealed a small positive effect $(\beta=.09, p=.013)$. However, this effect was smaller than the $\operatorname{MDE}(\beta=|.10|)$ which is why the present study does not have enough power to meaningfully interpret it. Thus, H10 is rejected. Together, the variables explained $62 \%$ of the variance of participants' intention to use the app $\left(R^{2}=\right.$ .62). Hypothesis H11 was supported, revealing a small negative association between knowledge about the app and privacy concerns $(\beta=-.15, p<.001)$. Forty-seven percent of the variance of privacy concerns was explained in the model $\left(R^{2}=.47\right)$. Although there was a significant positive relation between knowledge and perceived benefits $(\beta=.04, p=.035)$, it is too small to be meaningfully interpreted. Consequently, $\mathrm{H} 12$ had to be rejected. The variance of benefit perception was explained by $67 \%\left(R^{2}=\right.$ .67). Finally, knowledge about the contact-tracing app and trust in the app developers was positively correlated $(r=.52, p<.001)$ supporting H13. We also allowed the residuals of privacy concerns and perceived benefits to covary which revealed a positive relationship $(r=.11, p=.009)$.

In addition to the direct effects, we also analyzed the total (i.e., direct plus indirect) effects of knowledge and trust on future usage intention. There was a small positive total relationship between knowledge and usage intention $(\beta=.13, p=.002)$ and a large positive total relationship between trust and the intention to use the app in the future $(\beta=.63, p<.001)$.

\section{Multicollinearity}

Because there were very high relationships between trust and perceived benefits, an additional test on multicollinearity was performed. Results did not imply severe problems with multicollinearity. The additional analysis is available in the OSF material (https://osf.io/turbs/; Meier et al., 2021).

\section{Discussion}

The present study aimed to investigate psychological factors that impact the use of the German COVID-19 contact-tracing app by surveying a representative (concerning age and gender) German sample. Relationships of privacy concerns, perceived COVID-19 contact-tracing app benefits, trust in the app's privacy, and knowledge about the app were tested in two SEMs, one of them including actual app use and the other one future (continued) usage intention. The results of this work contribute to the general understanding of why people adopt or do not adopt helpful mobile applications in crisis situations and how mass acceptance of a necessary technology to manage a health crisis can best be achieved.

For actual app usage, results showed that participants who reported having privacy concerns did indeed less often use the tracing app than those who had fewer privacy concerns. In addition, persons who perceived the app to be beneficial were considerably more likely to use it than someone who perceived less benefits. 
Figure 2

Structural Equation Model With App Usage Intention as Endogenous Variable

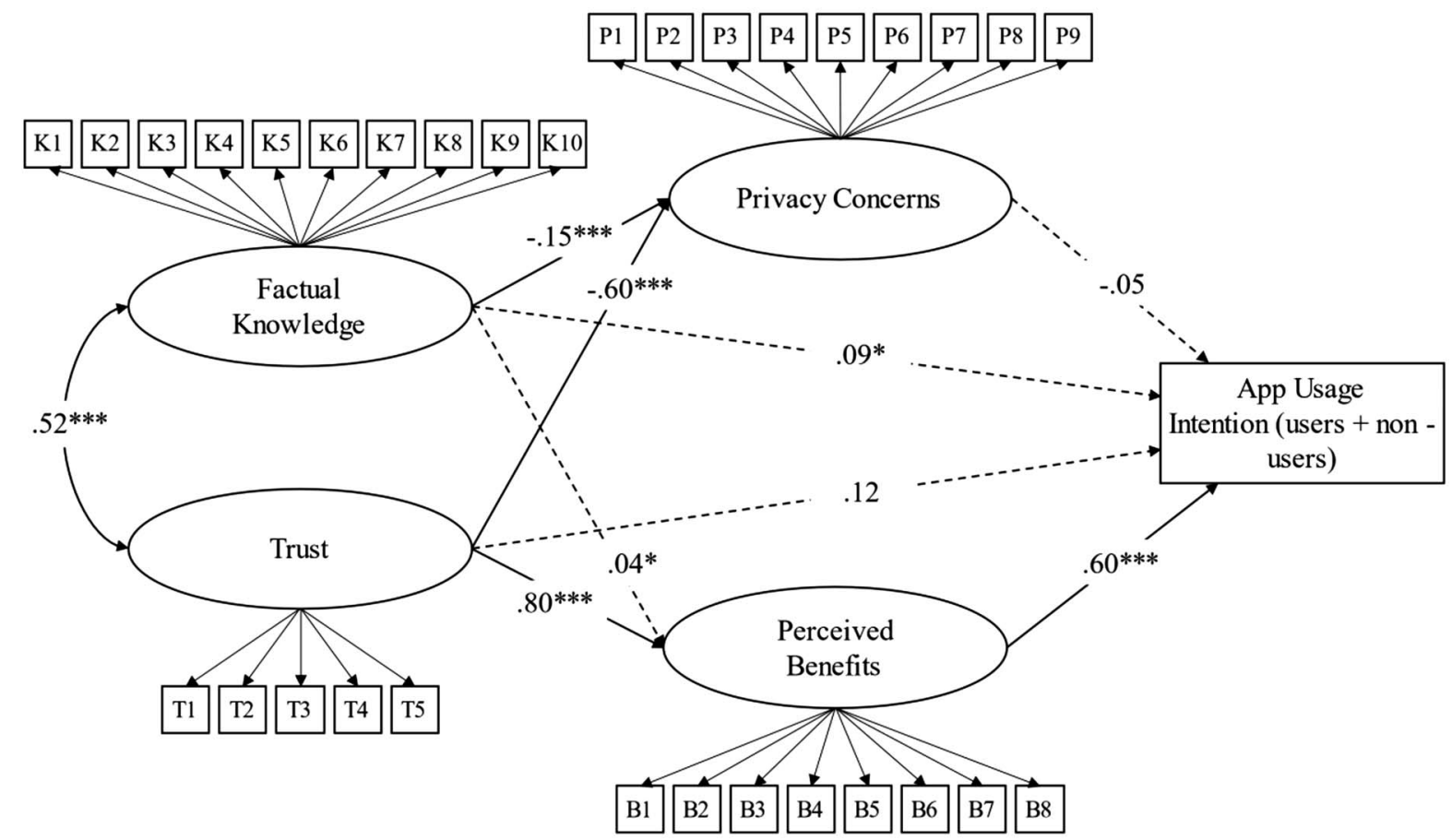

Note. Numbers represent standardized regression coefficients. Dotted lines indicate that the path is either statistically meaningless (not significant) or theoretically meaningless (below $\beta=1.101$ ).

$* * * p<.001 . * p<.05$.

Concerning trust, results did not reveal a significant direct relationship to app adoption (however, trust played a crucial indirect role; see below). Finally, people who knew more about the functionality of the app including its privacy-preserving nature did also more often install it. These findings indicate that privacy concerns, perceived benefits, and knowledge about the app played important roles in the adoption process of the German COVID-19 contacttracing app. It can be concluded that the perception of the app's benefits in containing the pandemic was the most important factor for the decision to use the app or not. However, also privacy relevant questions mattered. While privacy concerns seemed to play rather a minor role, those who had higher factual knowledge about how the app operates and that it is highly privacy-friendly were also more likely to use it. Hence, it can be argued that since both potential positive effects to the society as well as potential negative effects for personal privacy played a role in the actual adoption of the app, a privacy calculus seemed to play a role in deciding to download the app or not (cf. Culnan \& Armstrong, 1999). These findings are in line with prior privacy calculus studies also showing that benefit perceptions are more strongly related to information-sharing intentions than the perceptions of privacy costs (Bol et al., 2018) or privacy concerns (Dienlin \& Metzger, 2016). In contrast to these studies, the difference of the effect sizes in the present study was even higher. This is, at first sight, surprising as the individual user does not directly benefit from using the app, for instance, in the sense that it precludes infection with the virus. The benefits that arise by actively using the app are contributing to the common good and protecting others, rather than protecting oneself. Quite the contrary, it can even produce costs to the individual as it leads to the obligation to have oneself tested and to retreat to quarantine. Therefore, the distal benefits lie in the contribution to the societal goal to interrupt infection chains and to save lives. In sum, this allows for the optimistic conclusion that people would willingly trade their privacy for "a higher good," that is saving others' lives throughout the time of the pandemic - and that this willingness might be more pronounced than the willingness to trade privacy for personal, hedonic benefits when, for instance, using SNSs. It must be noted that the small relationship between privacy concerns and app use may be traced back to the fact that this app actually did not have any privacy issues. To our knowledge, this is the first study which gives insights into potential reasons for deciding pro or contra actual adoption of an existing COVID-19 app. Because we started the study 1 week after the app's launch, it may well be that not all persons who intended to actually download and use it already did so. Hence, it was possible that actual usage and future intention to use the application differed. To find out more about psychological processes that were related to usage intention as well as relations among the independent measures, the second model was computed.

With a view to the usage intention of the German COVID-19 contact-tracing app, we assumed that all variables would be directly related to participants' intention to use the app in the future. In addition, it was hypothesized that trust and knowledge about the app would be associated with privacy concerns and the perception of app benefits. Interestingly, regarding future intentions to use the contact- 
tracing app, we found privacy concerns to be unrelated to usage intentions. However, the perception of benefits was strongly associated with future usage intentions. This finding may be explained by the argument that privacy concerns more strongly affect a current action compared to an action in the future. This argumentation is consistent with the construal-level theory (Trope \& Liberman, 2010) that assumes near-future and distant-future events to differ in their mental representation. Hence, privacy concerns seemed to make actual usage more unlikely but did not play a significant role in people's consideration to probably use it or continue to use it. Benefits, in contrast, can be considered as the main reason for usage and it makes sense that they are positively related to both usage behavior and intention. Trust was not found to be related to participants' future usage intention directly. However, trust was strongly negatively related to participants' privacy concerns and strongly positively to the perception of app benefits. Thus, trust that the application is privacy preserving seems to play a crucial indirect role in the adoption of the contact-tracing app by substantially contributing to variables that directly influence usage intention. This finding is in line with previous studies, showing that trust can work as a risk mitigator (Krasnova et al., 2010) and can contribute to one's perception of usefulness of an application (Beldad \& Hegner, 2018). Besides trust, knowledge about the app's functioning was hypothesized to impact privacy concerns, perceived benefits, and usage intention. Results showed that respondents who had higher knowledge about the app were not more inclined to (continue to) use it. Moreover, factual knowledge was negatively related to privacy concerns but there was no relation between knowledge and benefit perception. This relation was hypothesized since former studies found that the situational perception of having online privacy can increase the perception of self-disclosure benefit (Meier, Schäwel, \& Krämer, 2020). In the present study, however, knowing that the COVID-19 contact-tracing app is a privacy-preserving approach did not relate to the perception of app advantages. This can probably be traced back to the specific form of operationalization here: While knowledge clearly centered around knowledge regarding privacy features, benefits of the app solely addressed its helpfulness in containing the pandemic. Finally, trust and knowledge were highly positively correlated implying that gaining more knowledge about how the app works can heighten one's trust toward its developers. However, a reversed direction is equally likely in the sense of blind trust: Participants who did not have exact knowledge about the app's functioning but trusted that the app will respect their privacy might have answered the knowledge questions accordingly and accidentally correctly. In sum, the role of trust and knowledge as they were found in the present study strengthens the claim that transparently communicating about the app's functions, working, and development contributes to users' trust and proficiency, and thereby decreases concerns and increases perceived benefits.

It must be stated that the factors investigated in the present study were not exhaustive. We particularly focused on the question whether different privacy-related variables played a role in adopting the German COVID-19 contact-tracing app. However, from other fields of research (e.g., technology acceptance model), we know that there are further factors impacting the adoption of new technologies (e.g., perceived ease of use). Still, the theoretical lens of this article was primarily put on a privacy calculus perspective. The portions of explained variance in both usage and usage intention were very high indicating that the variables played a significant role in app adoption and considerations of future use.

From a practical viewpoint, the results call for a stronger emphasis of the benefits coming along with the usage of the COVID-19 contract-tracing apps within the communication activities of politicians, media, and health institutions to encourage more individuals to adopt and actively use the app. It must be noted that this recommendation is only applicable for the German COVID-19 app that does not have any privacy issues. In general, we believe that it is indispensable to transparently disclose all potential privacy issues, so that users can engage in a fully informed cost-benefit analysis. Regarding theoretical implications, particularly the findings on the influential role of benefits revealed that not only the perception of individual advantages (such as receiving a notification in cases of contact with an infected person) contributes to the adoption of the contract-tracing app but also assets that apply to the society such as efficiently identifying infections patterns to interrupt them and protect others from COVID-19 infections. This extends prior findings for instance on self-disclosure on SNSs due to perceived individual benefits. Broadly speaking, benefits as they are understood in the privacy calculus can obviously also exist in the form of altruistic advantages.

\section{Limitations and Future Directions}

Some limitations of the present work should be mentioned. We did not ask participants whether their smartphone version was up-todate enough to install the app. As this is only true for approximately $82 \%$ of German smartphone users (Becker, 2020), one important reason for not using the app was disregarded. Because of the crosssectional design of the study, no causal conclusions can be drawn and some of the effects may be of reciprocal nature. Thus, future studies should address longitudinal effects. Further, the results of this study are only valid for the German population and the German COVID-19 contact-tracing app. Hence, care should be taken when transferring the results to other countries or cultures. Future studies may also want to consider cross-national samples and different contact-tracing apps. Another issue concerns some kind of redundancy of both models. Participants who used the app were much more likely to indicate a high usage intention and those who did not use it rather indicated a low future usage intention. Moreover, we combined two variables that are actually measuring slightly different things (i.e., usage intentions of users and nonusers) into one score. Although we did not find differences when separating both intentions (see OSF; Meier et al., 2021), it cannot be ruled out that the intention to continue to use an app differs from the intention to initially adopt an app. Another limitation relates to the possibility of multicollinearity. Although we checked for multicollinearity, it is still possible that the results of the models are slightly biased, as in some cases there were very high relations. This should be taken into account when interpreting the results. Finally, the results of the study were probably influenced by media and interindividual debates in Germany in the week following the launch of the app.

\section{Conclusion}

The unique strength of the present study is that, compared to prior work on COVID-19 apps, it does not rely on hypothetical apps but that it assesses participants' attitudes and perceptions of the German 
COVID-19 contact-tracing app shortly after its release thereby gathering more conclusive data on the influencing factors regarding tracing app usage during pandemics. Altogether, the findings indicate that both knowledge about the app and trust toward its developers are important elements in adopting the German COVID-19 contact-tracing app. Persons with higher knowledge and trust had reduced privacy concerns and those who trusted the app developers perceived much higher app advantages. Most remarkable, however, was the size of the effect of the relationship between perceived benefits and app adoption and usage intention, respectively. Thus, persons who perceived the app to be beneficial in containing the virus disease were much more likely to use it while those having privacy concerns were only slightly less likely to use it. Strikingly, the role of perceived benefits was even more pronounced compared to its role, for instance, in the context of self-disclosure on SNSseven though the benefits are merely distal and pertain to society rather than the individual. Based on the findings of this study, it can be recommended that the benefits of contact-tracing apps must be made clearly visible for people but that, in addition, measures must be taken to strengthen people's knowledge about all potential privacy threats which enables people to make a self-determined decision whether to adopt a contact-tracing app or not.

\section{References}

Altmann, S., Milsom, L., Zillessen, H., Blasone, R., Gerdon, F., Bach, R., Kreuter, F., Nosenzo, D., Toussaert, S., \& Abeler, J. (2020). Acceptability of app-based contact tracing for COVID-19: Cross-country survey study. JMIR mHealth and uHealth, 8(8), Article e19857. https://doi.org/10.2196/19857

Becker, K. B. (2020, June 18). So viele können die Corona-App überhaupt nutzen. Frankfurter Allgemeine Zeitung. https://www.faz.net/aktuell/ politik/inland/so-viele-deutsche-koennen-die-corona-app-ueberhauptnutzen-16819819.html

Beldad, A. D., \& Hegner, S. M. (2018). Expanding the technology acceptance model with the inclusion of trust, social influence, and health valuation to determine the predictors of German users' willingness to continue using a fitness app: A structural equation modeling approach. International Journal of Human-Computer Interaction, 34(9), 882-893. https://doi.org/10.1080/10447318.2017.1403220

Bhattacharya, R., Devinney, T. M., \& Pillutla, M. M. (1998). A formal model of trust based on outcomes. Academy of Management Review, 23(3), 459472. https://doi.org/10.5465/amr.1998.926621

Bol, N., Dienlin, T., Kruikemeier, S., Sax, M., Boerman, S. C., Strycharz, J., Helberger, N., \& de Vreese, C. H. (2018). Understanding the effects of personalization as a privacy calculus: Analyzing self-disclosure across health, news, and commerce contexts. Journal of Computer-Mediated Communication, 23(6), 370-388. https://doi.org/10.1093/jcmc/zmy020

Broadberry, S., \& Harrison, M. (2005). The economics of World War I: an overview. In S. Broadberry \& M. Harrison (Eds.), The economics of World War I (pp. 3-40). Cambridge University Press. https://doi.org/10.1017/ CBO9780511497339.002

Chan, E. Y., \& Saqib, N. U. (2021). Privacy concerns can explain unwillingness to download and use contact tracing apps when COVID-19 concerns are high. Computers in Human Behavior, 119, Article 106718. https://doi.org/10.1016/j.chb.2021.106718

Culnan, M. J., \& Armstrong, P. K. (1999). Information privacy concerns, procedural fairness, and impersonal trust: An empirical investigation. Organization Science, 10(1), 104-115. https://doi.org/10.1287/orsc.10 .1 .104

Curran-Everett, D. (2017). CORP: Minimizing the chances of false positives and false negatives. Journal of Applied Physiology, 122(1), 91-95. https:// doi.org/10.1152/japplphysiol.00937.2016
Dienlin, T., \& Metzger, M. J. (2016). An extended privacy calculus model for SNSs: Analyzing self-disclosure and self-withdrawal in a representative US sample. Journal of Computer-Mediated Communication, 21(5), 368-383. https://doi.org/10.1111/jcc4.12163

Dinev, T., \& Hart, P. (2006). An extended privacy calculus model for ecommerce transactions. Information Systems Research, 17(1), 61-80. https://doi.org/10.1287/isre.1060.0080

Djimeu, E. W., \& Houndolo, D. G. (2016). Power calculation for causal inference in social science: Sample size and minimum detectable effect determination. Journal of Development Effectiveness, 8(4), 508-527. https://doi.org/10.1080/19439342.2016.1244555

Eling, N., Krasnova, H., Widjaja, T., \& Buxmann, P. (2013). Will you accept an app? Empirical investigation of the decisional calculus behind the adoption of applications on Facebook [Conference session]. ICIS 2013 Proceedings: Security and privacy of information and IS, Milan, Italy.

Ferretti, L., Wymant, C., Kendall, M., Zhao, L., Nurtay, A., Abeler-Dörner, L., Parker, M., Bonsall, D., \& Fraser, C. (2020). Quantifying SARS-CoV2 transmission suggests epidemic control with digital contact tracing. Science, 368(6491), Article eabb6936. https://doi.org/10.1126/science .abb6936

Hassandoust, F., Akhlaghpour, S., \& Johnston, A. C. (2021). Individuals privacy concerns and adoption of contact tracing mobile applications in a pandemic: A situational privacy calculus perspective. Journal of the American Medical Informatics Association, 28(3), 463-471. https:// doi.org/10.1093/jamia/ocaa240

Horn, D. (2020, June 16). Wie sicher ist die Corona-Warn-App? Tagesschau. https://www.tagesschau.de/inland/corona-app-kritik-sicherheit-101.html

Johnson, N. P. A. S., \& Mueller, J. (2002). Updating the accounts: Global mortality of the 1918-1920 "Spanish" influenza pandemic. Bulletin of the History of Medicine, 76(1), 105-115. https://doi.org/10.1353/bhm .2002 .0022

Kaptchuk, G., Goldstein, D. G., Hargittai, E., Hofman, J. M., \& Redmiles, E. M. (2020). How good is good enough for COVID19 apps? The influence of benefits, accuracy, and privacy on willingness to adopt. arXiv. https://arxiv.org/abs/2005.04343

Keith, M. J., Babb, J., Furner, C., Abdullat, A., \& Lowry, P. B. (2016). Limited information and quick decisions: Consumer privacy calculus for mobile applications. AIS Transactions on Human-Computer Interaction, 8(3), 88-130. https://doi.org/10.17705/1thci.00081

Khan, G. F., Swar, B., \& Lee, S. K. (2014). Social media risks and benefits: A public sector perspective. Social Science Computer Review, 32(5), 606627. https://doi.org/10.1177/0894439314524701

Kostka, G., \& Habich-Sobiegalla, S. (2020). In times of crisis: public perceptions towards COVID-19 contact tracing apps in China, Germany and the US. SSRN Electronic Journal. https://doi.org/10.2139/ssrn .3693783

Krasnova, H., Eling, N., Abramova, O., \& Buxmann, P. (2014). Dangers of 'Facebook Login' for mobile apps: Is there a price tag for social information? [Conference session]. ICIS 2014 Proceedings: IS security and privacy, Auckland, New Zealand.

Krasnova, H., Spiekermann, S., Koroleva, K., \& Hildebrand, T. (2010). Online social networks: Why we disclose. Journal of Information Technology, 25(2), 109-125. https://doi.org/10.1057/jit.2010.6

Lakens, D. (2021). Sample size justification. PsyArXiv. https://doi.org/10 31234/osf.io/9d3yf

Lin, L., \& Hou, Z. (2020). Combat COVID-19 with artificial intelligence and big data. Journal of Travel Medicine, 27(5), 1-4. https://doi.org/10.1093/ $\mathrm{jtm} / \mathrm{taaa} 080$

Meier, Y., Meinert, J., \& Krämer, N. (2021, June 4). Investigating factors that affect the adoption of Covid-19 contact-tracing apps. A privacy calculus perspective. https://doi.org/10.17605/OSF.IO/TURBS

Meier, Y., Schäwel, J., \& Krämer, N. C. (2020). The shorter the better? Effects of privacy policy length on online privacy decision-making. Media 
and Communication, 8(2), 291-301. https://doi.org/10.17645/mac v8i2.2846

Meier, Y., Schäwel, J., Kyewski, E., \& Krämer, N. C. (2020). Applying protection motivation theory to predict facebook users' withdrawal and disclosure intentions [Conference session]. Proceedings of the 11th International Conference on Social Media and Society, Toronto, ON, Canada. https://doi.org/10.1145/3400806.3400810

Mello, M. M., \& Wang, C. J. (2020). Ethics and governance for digital disease surveillance. Science, 368(6494), 951-954. https://doi.org/10 $.1126 /$ science.abb 9045

Podsakoff, P. M., MacKenzie, S. B., \& Podsakoff, N. P. (2012). Sources of method bias in social science research and recommendations on how to control it. Annual Review of Psychology, 63(1), 539-569. https://doi.org/ 10.1146/annurev-psych-120710-100452

Princi, E., \& Krämer, N. C. (2020). Out of control - Privacy calculus and the effect of perceived control and moral considerations on the usage of IoT healthcare devices. Frontiers in Psychology, 11, Article 582054. https:// doi.org/10.3389/fpsyg.2020.582054

Trang, S., Trenz, M., Weiger, W. H., Tarafdar, M., \& Cheung, C. M. K. (2020). One app to trace them all? Examining app specifications for mass acceptance of contact-tracing apps. European Journal of Information Systems, 29(4), 415-428. https://doi.org/10.1080/0960085X.2020 .1784046

Trepte, S., Teutsch, D., Masur, P. K., Eicher, C., Fischer, M., Hennhöfer, A., $\&$ Lind, F. (2015). Do people know about privacy and data protection strategies? Towards the "Online Privacy Literacy Scale" (OPLIS). Reforming European data protection law (pp. 333-365). Springer. https:// doi.org/10.1007/978-94-017-9385-8_14

Trope, Y., \& Liberman, N. (2010). Construal-level theory of psychological distance. Psychological Review, 117(2), 440-463. https://doi.org/10.1037/ a0018963
Utz, S., \& Krämer, N. C. (2009). The privacy paradox on social network sites revisited: The role of individual characteristics and group norms. Cyberpsychology: Journal of Psychosocial Research of Cyberspace, 3(2), 2. https://cyberpsychology.eu/article/view/4223/3265

Wang, Y. A., \& Rhemtulla, M. (2021). Power analysis for parameter estimation in structural equation modeling: A discussion and tutorial. Advances in Methods and Practices in Psychological Science, 4(1), 1-17. https://doi.org/10.1177/2515245920918253

Walrave, M., Waeterloos, C., \& Ponnet, K. (2020). Adoption of a contact tracing app for containing COVID-19: A health belief model approach. JMIR Public Health and Surveillance, 6(3), Article e20572. https:// doi.org/10.2196/20572

World Health Organization. (2020, September 17). Coronavirus disease (COVID-19) advice for the public. https://www.who.int/emergencies/ diseases/novel-coronavirus-2019/advice-for-public

Xia, Y., \& Yang, Y. (2019). RMSEA, CFI, and TLI in structural equation modeling with ordered categorical data: The story they tell depends on the estimation methods. Behavior Research Methods, 51(1), 409-428. https:// doi.org/10.3758/s13428-018-1055-2

Yasaka, T. M., Lehrich, B. M., \& Sahyouni, R. (2020). Peer-to-peer contact tracing: Development of a privacy-preserving smartphone app. JMIR mHealth and uHealth, 8(4), Article e18936. https://doi.org/10 2196/18936

Zheng, S., Apthorpe, N., Chetty, M., \& Feamster, N. (2018). User perceptions of smart home IoT privacy. Proceedings of the ACM on HumanComputer Interaction, 2(CSCW), 200. https://doi.org/10.1145/327446

Received December 23, 2020

Revision received June 4, 2021

Accepted June 6, 2021 